TECHNIQUES OF REMOVAL

There are several methods described to facilitate removal. ${ }^{23}$

The larva may be removed by enlarging the central punctum with a cruciate incision under local anaesthetic, grasping the larva with forceps, and teasing it out. Care must be taken not to damage the larva and so risk leaving parts behind in the wound, as this may result in a local foreign body reaction. This is the technique we successfully used.

Alternative methods rely on the fact that the larvae are obligate aerobes. Thus they can be coaxed into the open by occluding their air supply with adhesive tape, Vaseline, heavy makeup cream, or immersion of the affected part in oil. This results in the larvae struggling to the surface, when removal can be completed by grasping it with a fine forceps. Perhaps the most novel method of removal described is "bacon therapy". ${ }^{4}$ Multiple strips of raw bacon are placed over the nodule, with the fat occluding the central punctum. The larva migrates into the bacon over a period of hours sufficiently to allow its intact removal with forceps. These latter techniques minimise scarring. After removal of the larva, the lesions usually heal spontaneously without the need for routine antibiotic cover

\section{Comment}

As areas in south and central America become more and more popular as tourist destinations, infestations such as these with Dermatobia hominis which have long larval stages will present more frequently to $A \& E$ departments in the United Kingdom. They are often misdiagnosed as cellulitis, furunculosis, or infected sebaceous cysts. Diagnosis should be considered if there is a history of exposure and suggestive physical findings, as outlined above. Once the diagnosis is made, removal can be accomplished by the methods outlined.

We would like to thank Dr Richard Jennings, The Hospital of Tropical Diseases, London for valuable advice and assistance.

Manson-Bahr PEC, Bell DR. Manson's tropical diseases, 19th ed. London: Bailliere Tindall, 1987:1463-5.

Rooney S, Kerrigan D. Bot fly bite. Postgrad Med J 1993;69:411-3

Nunzi E Rogioletti F, Rebora A Removal of Dermatobia hominis larvae. Arch Dermatol 1986;122:140.

hominis larva. Arch Dermatol 1986;

E, Felsenstein D. Bacon therapy and furuncular myiasis. JAMA 1993;270:2087-8.

\title{
A case of muscle abscess presenting to an accident and emergency department
}

\section{Accident and \\ Emergency \\ Department, \\ Derriford Hospital, \\ Plymouth PL6 8DH \\ M A Howell \\ H R Guly \\ Correspondence to: \\ Mr M A Howell, Accident \\ and Emergency Department, \\ Bristol Royal Infirmary, \\ Marlborough Street, Bristol \\ BS2 8HW.}

Accepted for publication

13 November 1996

\begin{abstract}
A case is reported of a patient with acute primary muscle abscess who presented to the accident and emergency department with hip pain. Pyomyositis must be considered as a cause of muscle pain especially around the hip. A brief discussion of the diagnosis and management of pyomyositis is also presented.

(F Accid Emerg Med 1997;14:180-182)
\end{abstract}

Keywords: pyomyositis; hip pain

Accident and emergency (A\&E) departments often see patients with acute musculoskeletal pain. In this paper we present a case of acute muscle abscess (pyomyositis) presenting to an $A \& E$ department with hip pain and we briefly review the published reports on muscle abscesses.

\section{Case report}

A 46 year old previously healthy civil servant presented to the $A \& E$ department with a four day history of right hip and groin pain which had come on after playing table tennis. Rest and simple analgesics had been ineffective. On examination he had a temperature of $37.6^{\circ} \mathrm{C}$ and a tender right hip and pubic symphysis, with a reduced range of movement in the hip and inability to weight bear. Radiographs of the hip were normal, as was an ultrasound scan. A full blood count was unremarkable but the erythrocyte sedimentation rate (ESR) was increased at $32 \mathrm{~mm} / \mathrm{h}$. Blood cultures were taken and the patient admitted under the care of the A\&E team for rest and analgesia.

The blood cultures grew Staphylococcus aureus so flucloxacillin $2 \mathrm{~g}$ four times daily was started. The general condition of the patient improved but his reduced hip movement persisted, as did his pyrexia. The possibility of a psoas abscess was considered and a further ultrasound scan was performed four days after admission. This showed a $6 \mathrm{~cm}$ mass inferomedial to the right inguinal ligament, thought by the radiologist to be a strangulated hernia though the general surgeons did not think he had a hernia clinically. A computerised tomography (CT) scan the same day showed an inflamed right adductor brevis muscle. Liver function tests at this stage were deranged, and the patient was thought to be septicaemic. A 

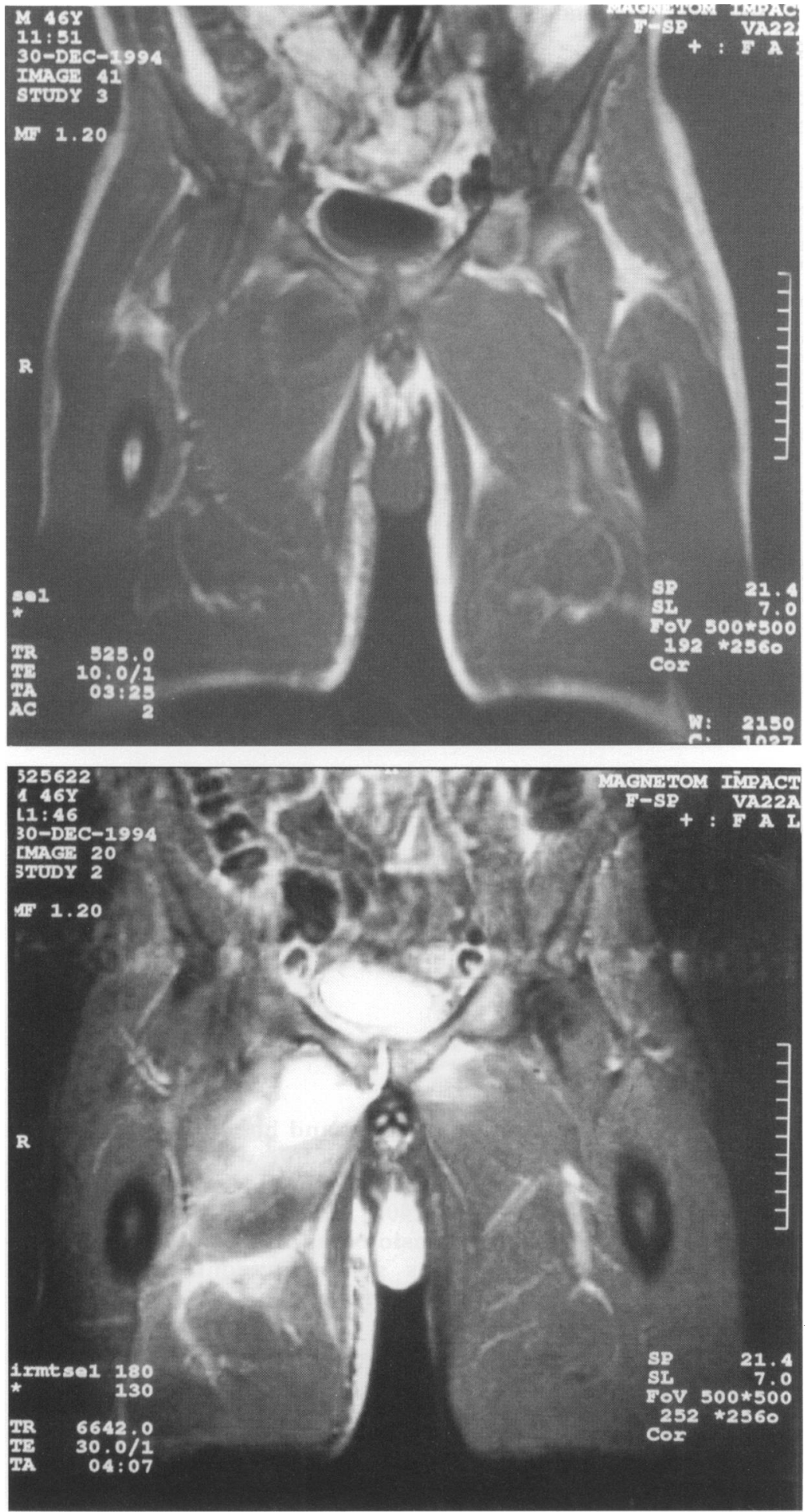

Figure 1 MRI scan showing the abscess of the patient's right hip.

magnetic resonance imaging (MRI) scan on the following day (fig 1) showed an abscess in obturator externus, adductor magnus, and adductor brevis, with surrounding oedema and distortion of muscle groups. The abscess was aspirated under ultrasound guidance: $10 \mathrm{ml}$ of thick pus were removed and a catheter left in the abscess cavity for seven days. The pus subsequently grew staphylococci with the same sensitivities as that from the original blood cultures.

The patient completed a four week course of flucloxacillin and made a complete recovery.

\section{Discussion}

Acute primary muscle abscess or pyomyositis is rare in Western countries ${ }^{12}$ and its diagnosis often delayed. It is much more prevalent in the tropics. ${ }^{34}$ Pyomyositis is relatively more common in children ${ }^{5}$ and has an equal sex distribution.

The aetiology of acute muscle abscess is believed to involve trauma, which is often trivial. Subsequent bacteraemia enables bacteria to infect this injured muscle. However, minor muscle trauma is common, whereas pyomyositis is rare. Diabetes mellitus, ${ }^{6}$ steroid treatment, and immunodeficiency states including HIV infection ${ }^{7}$ have been identified as predisposing factors. Muscle abscess secondary to intramuscular injections has also been reported. $^{8}$

The most common muscles affected are around the hip, including the psoas, piriformis, obturators, adductors, glutei, and quadriceps. Psoas abscesses can also occur secondary to bowel perforation or spinal infection. Upper limb muscles and even neck muscles are occasionally implicated. ${ }^{910}$ The commonest infecting organisms in tropical climates are mycobacteria, ${ }^{11}$ hydatid, ${ }^{12}$ and nocardia ${ }^{13}$ species, whereas in temperate zones the main pathogens are staphylococci, ${ }^{14}$ streptococci, ${ }^{15}$ haemophilus, and coliforms.

Diagnosis is difficult and the differential diagnoses are legion, including muscle strain, synovitis, tumour, osteomyelitis, septic arthritis, Perthe disease, rheumatoid arthritis, slipped upper femoral epiphysis, strangulated hernia, venous thrombosis, and thrombophlebitis. Apart from a history of trauma, often minor, there may be non-specific symptoms such as aches, swelling, chills, and night sweats. Physical findings include local tenderness, masses, erythema, and low grade fever, together with reduced movements of joints acted upon by the affected muscles, most commonly the hip. Complications may occur, particularly peripheral nerve palsies. ${ }^{1617}$

Laboratory investigations typically show a leucocytosis and left shift together with raised ESR and C reactive protein. Blood cultures are usually positive and liver function tests deranged if septicaemia is present.

Radiological investigations are the mainstay of diagnosis and treatment planning. ${ }^{18}$ Plain radiographs may show a soft tissue mass or shadow; ultrasound scanning may reveal an abscess. Radionuclide white cell or gallium scans show increased uptake and may occasionally specifically show an abscess. ${ }^{19}$ CT scanning may show a mass, localised attenuation, and ring enhancement with intravenous contrast. $^{20}$ MRI with gadolinium enhancement $^{2021}$ is probably the investigation of choice at present.

The treatment of pyomyositis is drainage of the muscle abscess, often under ultrasound or CT guidance, together with antibiotics selected on the basis of cultures and antibiotic sensitivities. With early diagnosis and treatment, prognosis is excellent and full recovery is normal. Delay in diagnosis and therefore in 
treatment can lead to disseminated disease and systemic complications. ${ }^{22}$

Primary muscle abscess is a rare condition but must be considered as a cause of muscle pain especially around the hip.

1 Christin L, Sarosi GA. Pyomyositis in North America: case reports and review. Clin Infect Dis 1992;15:668-77.

2 Hall RL, Callaghan JJ, Moloney E, Martinez S, Harrelson

JM. Pyomyositis in a temperate climate: presentation, diagnosis and treatment. J Bone Joint Surg (Am) 1990;72: nosis and

3 Steinig JP, Odom JW, Jurgenson PF, Williams JS. Tropical pyomyositis. J Med Assoc Georgia 1992;81:75-6.

4 Gambir IS, Singh DS, Gupta SS, Gupta PR, Kumar M Tropical pyomyositis in India: a clinico-histopathological study. J Trop Med Hyg 1992;95:42-6.

5 Renwick SE, Ritterbusch JF. Pyomyositis in children. J Pediatr Orthop 1993;13:769-72.

6 Brown RL. Pyomyositis in patients with diabetes. Com puted tomography as a key to diagnosis. Postgrad Med 1989;86:79-81.

7 Rodgers WB, Yodlowski ML, Mintzer CM. Pyomyositis in patients who have the human immunodeficiency virus. Case report and review of the literature. J Bone Joint Surg (Am) 1993;75:588-92.

8 Rygnestad T, Kvam AM. Streptococcal myositis and tissue necrosis with intramuscular administration of diclofenac (Voltaren). Acta Anaesthesiol Scand 1995;39:1128-30.

9 Michaels BM, Orgill DP, Santos AA. Tropical pyomyositis presenting in the upper extremity. Arch Surg 1995;130:
$446-7$.
10 Flory P, Brocq O, Euller-Ziegler L, Ziegler G. Pyomyositis: cervical localisation. J Rheumatol 1993;20:1411-3.

11 Huang DY. Tuberculous muscle abscess: an unusual presentation of tuberculosis. Am J Med 1990;88:57-9N.

12 Martin J, Marco V, Zidan A, Marco C. Hydatid disease of the soft tissues of the lower limb: findings in three cases. Skel Radiol 1993;22:511-4.

13 Gill MV, Cunha BA. Nocardia asteroides buttock abscess. Infection 1995;23:126-7.

14 Armstrong DG, D'Amato CR, Strong ML. Three cases of staphylococcal pyomyositis in adolescence, including one patient with neurologic compromise. J Pediatr Orthop 1993;13:452-5.

15 Peetermans WE, Buyse B, Vanhoof J. Pyogenic abscess of the gluteal muscle due to Streptococcus pneumoniae the gluteal muscle due to Streptococcus promment]. Clin Infect Dis 1993;17:939.

16 Chen WS. Sciatica due to piriformis pyomyositis. Report of a case. J Bone Joint Surg (Am) 1992;74:1546-8.

17 Pai VS, Yee E, Lawson D, Curtis G. Iliacus pyomyositis with involvement of lateral cutaneous nerve of the thigh. NZ involvement of lateral

18 Applegate GR, Cohen AJ. Pyomyositis: early detection utilizing multiple imaging modalities. Magn Reson Imag ing 1991;9:187-93.

19 Schiff RG, Silver L. Tropical pyomyositis. Demonstration of extent and distribution of disease by gallium scintigraphy. Clin Nuclear Med 1990;15:542-4.

20 Gordon BA, Martinez S, Collins AJ. Pyomyositis: characteristics at CT and MR imaging. Radiology 1995;197:27986.

21 Hopkins KL, Li KC, Bergman G. Gadolinium-DTPAenhanced magnetic resonance imaging of musculoskeletal infectious processes. Skel Radiol 1995;24:325-30.

22 McGill PE. Bacterial infections: pyomyositis. Baillieres Clin Rheumatol 1995;9:193-200.

\section{Malignant hypertension presenting as blurred vision in a 43 year old intravenous drug abuser}

\section{Department of \\ Ophthalmology, St \\ James' University \\ Hospital, Beckett \\ Street, Leeds LS9 7TF \\ G Walters}

T R Dabbs

Correspondence to: Mr G Walters.

Accepted for publication 6 February 1997

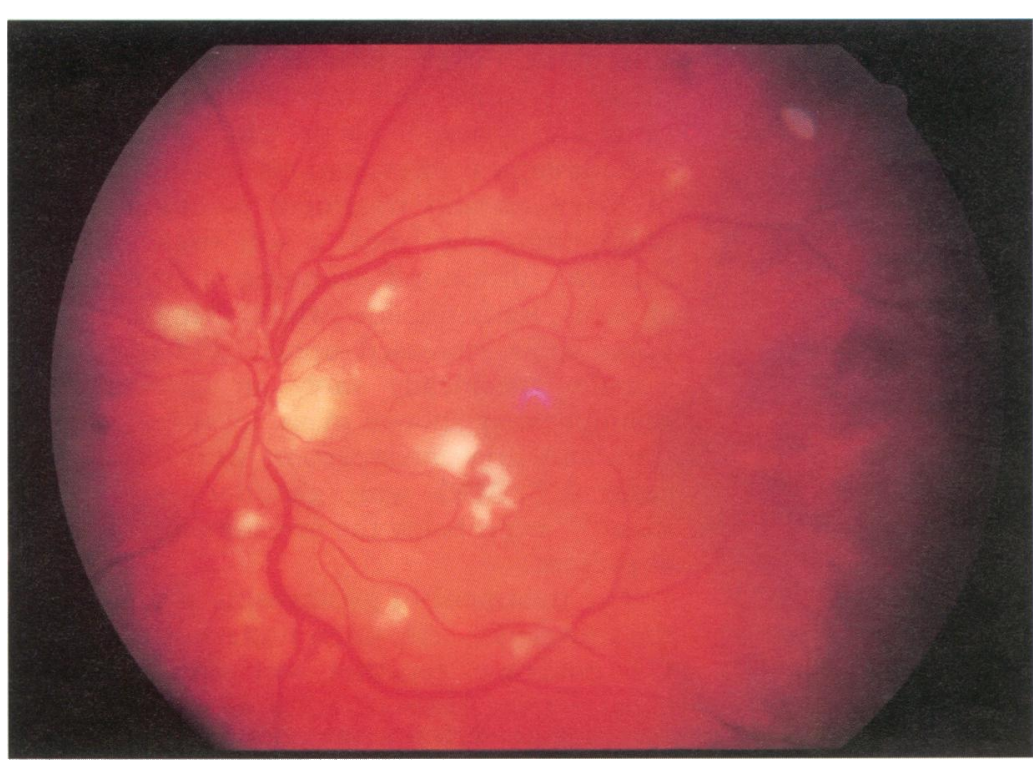

Figure 1 Retinal photograph of the left eye showing optic disc swelling, arteriolar attenuation, arteriovenous nipping, prominent cotton wool spots, and haemorrhages.

\begin{abstract}
A 43 year old intravenous drug abuser presented to the accident and emergency department with a three week history of bilateral visual loss and frontal headaches. Fundoscopy revealed bilateral retinal cot-
\end{abstract}

\section{importance of checking the blood pres- sure of all patients presenting with visual} loss.

(F Accid Emerg Med 1997;14:182-183)

Keywords: malignant hypertension; visual loss

\section{Case report}

A 43 year old man presented to the accident and emergency (A\&E) department with a three week history of bilateral blurred vision and frontal headaches. He was a long standing intravenous heroin abuser and attended because of difficulty in seeing to inject. In his past history he had received a brain contusion injury after a road traffic accident two years previously. He had recently been found to be hepatitis $\mathrm{C}$ positive, although he had not received any treatment for this. $\mathrm{He}$ was otherwise well.

On examination in the $\mathrm{A} \& \mathrm{E}$ department, his vision was found to be reduced to $6 / 24$ in the right eye and $6 / 36$ in the left eye (normal $6 / 6$ or 